\title{
The vesicular nucleotide transporter (VNUT) is involved in the extracellular ATP effect on neuronal differentiation
}

\author{
Aida Menéndez-Méndez ${ }^{1}$ - Juan Ignacio Díaz-Hernández ${ }^{1}$. \\ M. Teresa Miras-Portugal ${ }^{1}$
}

Received: 4 November 2014 / Accepted: 25 March 2015 /Published online: 7 April 2015

(C) The Author(s) 2015. This article is published with open access at Springerlink.com

\begin{abstract}
Before being released, nucleotides are stored in secretory vesicles through the vesicular nucleotide transporter (VNUT). Once released, extracellular ATP participates in neuronal differentiation processes. Thus, the expression of a functional VNUT could be an additional component of the purinergic system which regulates neuronal differentiation and axonal elongation. In vitro expression of VNUT decreases neuritogenesis in N2a cells differentiated by retinoic acid treatment, whereas silencing of VNUT expression increases the number and length of neurites in these cells. These results highlight the role of VNUT in the neuritogenic process because this transporter regulates the ATP content in neurosecretory vesicles.
\end{abstract}

Keywords VNUT $\cdot$ Neuritogenesis $\cdot$ Retinoic acid differentiation $\cdot$ ATP $\cdot$ Neuroblastoma cells $\cdot$ SLC17A9

\section{Abbreviations \\ VNUT Vesicular nucleotide transporter \\ RFP Red fluorescent protein}

Aida Menéndez-Méndez and Juan Ignacio Díaz-Hernández contributed equally to this work.

Electronic supplementary material The online version of this article (doi:10.1007/s11302-015-9449-4) contains supplementary material, which is available to authorized users.

Juan Ignacio Díaz-Hernández

jidiazhe@ucm.es

1 Facultad de Veterinaria, Departamento de Bioquímica y Biología Molecular IV, Universidad Complutense de Madrid, Madrid, Spain

\author{
GFP Green fluorescent protein \\ N2a Neuro-2a \\ shRNA Small hairpin RNA
}

\section{Introduction}

Neurotransmission and neuroendocrine signaling depends on the regulated release of a large variety of vesicular-stored signaling molecules. Non-peptidergic compounds require specific vesicular transporters, all of them are members of the solute carrier family (SLC) $[1,2]$. Nowadays, it is widely accepted that a single secretory vesicle contains more than one neurotransmitter and requires the presence of the corresponding specific transporters in the membrane vesicles. ATP and a large variety of nucleotidic compounds are among the most frequent substances co-stored with the classical neurotransmitters earlier discovered, such as acetylcoline, catecholamines, serotonine, and even glutamate, or other aminoacidic compounds $[3,4]$. To explain the complexity of signaling events upon vesicular release, G. Burnstock coined the term of co-transmission [5].

The abundant presence and distribution of secretory vesicles containing ATP and other nucleotides require the understanding of their transport and storage [6]. Regarding this, some previous work from our group contributed to the kinetic characterization of the vesicular nucleotide transporter (VNUT), showing its dependence on the $\mathrm{pH}$ gradient between vesicles lumen and cytosol, its prominent mnemonic behavior and the broad substrate specificity [7] . Recently, Moriyama's group cloned and identified VNUT as the product of the SLC17A9 gene [8]. Proteoliposomes reconstituted with the purified recombinant protein exhibit $\Delta \psi$-driven and $\mathrm{Cl}^{-}$dependent ATP transport. Expression in secretory tissues such as adrenal gland and suppression by small interfering RNA, 
together with the concomitant reduction on ATP release, confirmed the SLC17A9 as the VNUT transporter.

Once released, ATP and the other stored nucleotides act on their specific P2X ionotropic or P2Y metabotropic receptor families, located in the same or neighboring cells $[9,10]$. A large number of ectonucleotidases accomplish the enzymatic degradation of nucleotides to adenosine and inorganic phosphate, finishing their action on P2 receptors [11-13]. All the molecules with a role in these cellular processes are grouped under the name of purinome, and they are specific for different cell types and physiopathological situations, which require a convenient experimental model. Among these models, embryonic hippocampal neurons and neuroblastoma N2a cell line, where P2X7 receptors are functional and mainly located at the axonal growth cone, have been proved to be suitable to understand the sequence of purinergic events for axonal growth and branching [14-16]. The presence of extracellular ATP and its effect on P2X7 receptors reduces axonal growth through a signaling cascade requiring intracellular $\mathrm{Ca}^{2+}$ increase $[17,14]$. In contrast, either degradation of ATP by ectonucleotidases activity or antagonizing or silencing of $\mathrm{P} 2 \mathrm{X} 7$ receptors is able to increase axonal length [17]. However, the involvement of VNUT in the regulation of neural differentiation and axonal elongation remains unknown. In this report, we have analyzed the consequence of VNUT expression/silencing in the process of neuritogenesis that occurs in N2a cells after treatment with retinoic acid. Thus, VNUT expression decreases the number and length of neurites, and this effect is reversed by the silencing of VNUT expression. These results indicate that the extracellular presence of ATP is linked to its vesicular content and availability to be released, which directly involves the vesicular transporter VNUT in the neuritogenesis and differentiation processes.

\section{Materials and methods}

\section{Plasmid constructs and design of shRNAs}

Mouse VNUT (NM_183161) was amplified from IMAGE consortium bacterial clone (IMAGE clone 4986674) by PCR and ligated into the pcDNA3.1 $(+)$ /myc-His A vector (Life Technologies) generating VNUT flagged with c-myc epitope (VNUT-myc). pRFP-C-RS vector obtained from Origene was used as transfection control. VNUT knockdown was achieved by RNA interference using a vector-based small hairpin RNAs (shRNA) approach (pSUPER.neo.GFP, Oligoengine). Several shRNA targets for VNUT were designed according to a previously reported rational-design protocol [18] and sequence 5'-GAACAAGAAGGAGGCTGGTATCGTGCTC A-3' was selected. Specificity of the sequence was confirmed by a BLAST analysis for mouse VNUT. As a control, we used the firefly-luciferase-targeted oligonucleotide 5'-CTGACG
CGGAATACTTCGA-3'. Synthetic forward and reverse 64nucleotide oligonucleotides (Sigma-Aldrich) were designed, annealed, and inserted into BglII-HindIII sites of the pSUPER.neo.GFP vector following the manufacturer's instructions. These plasmid constructs express shRNA targeted against VNUT (shVNUT) or luciferase (non-targeting, shNT) transcripts. The concomitant expression of GFP from this vector allowed transfected cells to be identified by fluorescence.

\section{Cell culture}

N2a cells were cultured in Dulbecco's modified eagle medium (DMEM, Sigma-Aldrich) supplemented with Glutamax ${ }^{\circledR}$ (Invitrogen), penicillin/streptomycin (Invitrogen), and $10 \%$ heat-inactivated FBS (Gibco ${ }^{\circledR}$, Life Technologies). Cells were grown at $37^{\circ} \mathrm{C}$ in a humidified atmosphere containing $5 \%$ $\mathrm{CO}_{2}$. For differentiation studies, cells were seeded at a density of $10^{5}$ cells $/ \mathrm{cm}^{2}$ in low-serum medium (DMEM containing $0.5 \%$ FBS) supplemented with $10 \mu \mathrm{M}$ retinoic acid (SigmaAldrich), renewed every 2 days as described by Tremblay et al. (2010) [19]. The assays were performed on day five of differentiation.

\section{Transfections}

$\mathrm{N} 2$ a cells were plated at $8 \times 10^{5} \mathrm{cell} / \mathrm{cm}^{2}$ in 6 -well plates and transiently transfected with $2.5 \mu \mathrm{g}$ of the different plasmids using Lipofectamine ${ }^{\mathrm{TM}} 2000$ (Life Technologies) following the manufacturer's instructions. After $6 \mathrm{~h}$, the medium was removed and the cells were further incubated for the indicated time periods in DMEM supplemented with Glutamax ${ }^{\circledR}$ (Invitrogen), penicillin/streptomycin (Invitrogen), and $10 \%$ heat-inactivated FBS. In the case of co-transfections with two plasmids, $1.25 \mu \mathrm{g}$ was used for each plasmid in order not to exceed total DNA quantity.

\section{RT-PCR and quantitative real-time PCR}

Total RNA was purified from cultured N2a cells using a SpeedTools Total RNA Extraction kit (Biotools) according to the manufacturer's instructions. After digestion with TURBO DNase (Ambion), $1 \mu \mathrm{g}$ of total RNA was quantified and reversed transcribed using M-MLV reverse transcriptase, $6 \mu \mathrm{g}$ of random primers, and $350 \mu \mathrm{M}$ dNTPs (all from Invitrogen). The quantitative real-time PCR (qPCR) reaction were carried out using LuminoCt qPCR ReadyMix (Sigma-Aldrich), $5 \mu \mathrm{l}$ of the cDNA previously synthesized and $1.25 \mu \mathrm{l}$ of specific commercial oligonucleotide primers for mouse VNUT (TaqMan ${ }^{\circledR}$ Gene Expression Assay, Life Technologies Assay ID Mm00805914_m1), as well as for the housekeeping gene glyceraldehyde 3-phosphate dehydrogenase (GAPDH, TaqMan ${ }^{\circledR}$ Gene Expression Assay, Life TechnologiesAssay ID Mm99999915_g1) in $25 \mu$ final volume. The reaction 
Fig. 1 Characterization of molecular tools to study expression or silencing of VNUT expression in N2a cells. a Schematic representation of the construct used for expression of VNUT in N2a cells. b VNUT mRNA levels analyzed by qPCR of transfected N2 cells with VNUT-myc and either shNT or shVNUT. shNT was used as negative control. The values represent the mean $\pm \operatorname{SEM}(n=3$, $* * * P<0.001$, unpaired Student's $t$ test). $\mathbf{c}$ Western blotting of untransfected N2a cells or N2a cells transfected with either VNUT-myc and shNT or shVNUT. $\alpha$-Tubulin was used as internal loading control. Graph bars represent the mean \pm SEM $(n=3, * * P<0.01$, unpaired Student's $t$ test). d Representative confocal images showing immunostaining against c-myc epitope fused to VNUT (red) and synaptophysin (green) of N2a cells transfected with VNUTmyc. Nuclei are counterstained with DAPI (blue). Scale bar $10 \mu \mathrm{m}$. Inset represents $4 \times$ magnification of indicated cell. Arrows indicate the most prominent VNUT and synaptophysin positive vesicles. $5 \mu \mathrm{m}$ a

b

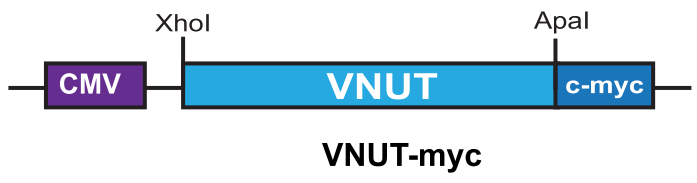

C
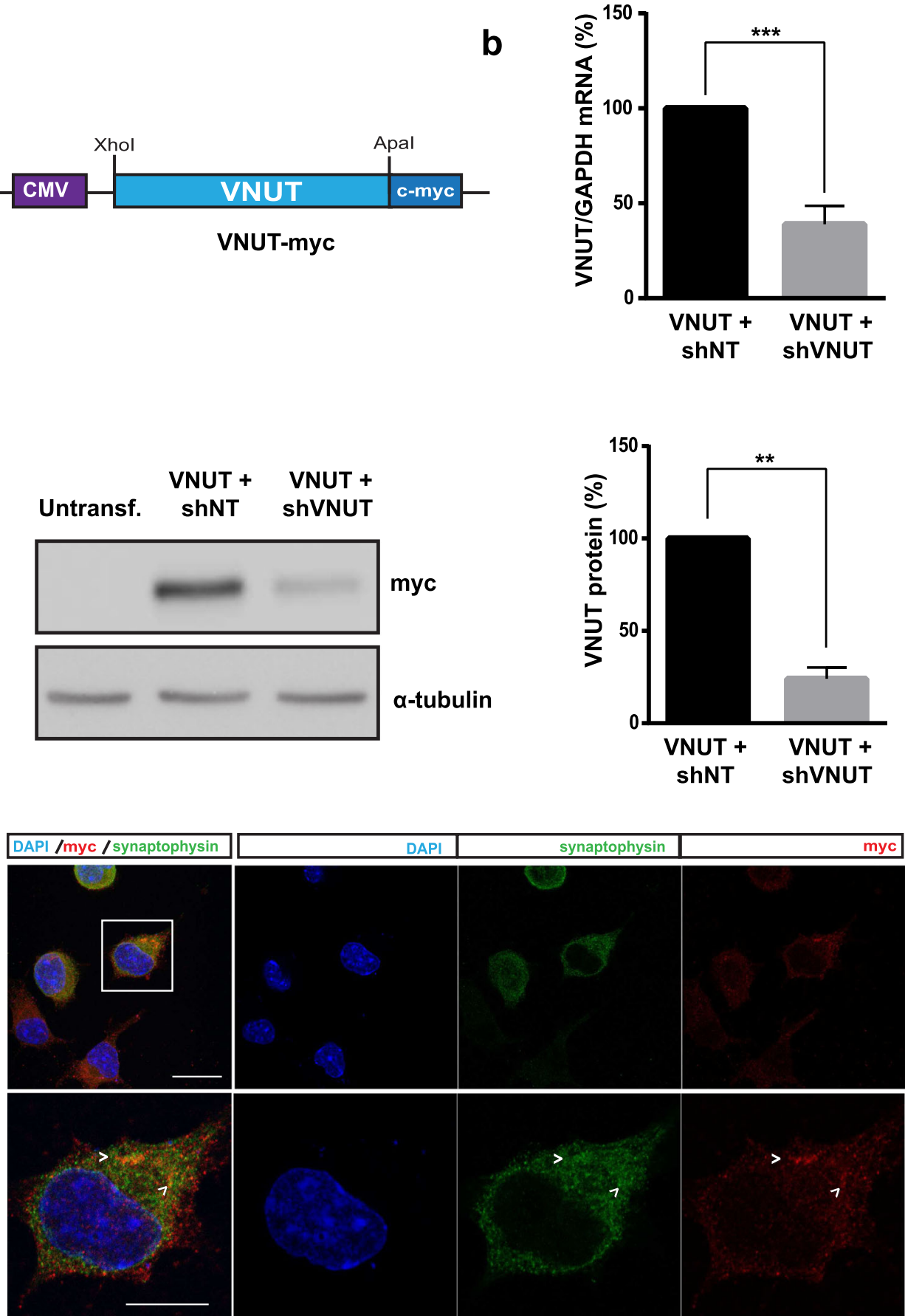

was realized in a StepOnePlus Real-Time PCR System (Applied Biosystems) as follows: denaturation, one cycle of $95^{\circ} \mathrm{C}$ for $20 \mathrm{~s}$, followed by 40 cycles each of $95^{\circ} \mathrm{C}$ for $1 \mathrm{~s}$ and $60^{\circ} \mathrm{C}$ for $20 \mathrm{~s}$. The results were normalized as indicated by parallel amplification of the endogenous housekeeping gene GAPDH.

\section{Western blotting}

$\mathrm{N} 2 \mathrm{a}$ cells were lysed and homogenized for $1 \mathrm{~h}$ at $4{ }^{\circ} \mathrm{C}$ in lysis buffer containing $50 \mathrm{mM}$ Tris/ $\mathrm{HCl}, 150 \mathrm{mM} \mathrm{NaCl}, 1 \%$
Nonidet P40 and Complete ${ }^{\mathrm{TM}}$ Protease Inhibitor Cocktail Tablets (Roche Diagnostics $\mathrm{GmbH}$ ), pH 7.4. Separation of the proteins was performed on $10 \%$ SDS-PAGE gels. Proteins were transferred to nitrocellulose membranes, blocked for $1 \mathrm{~h}$ at room temperature (RT) with $5 \%$ nonfat dried milk in tween-phosphate-buffered saline (137 mM NaCl, $2.7 \mathrm{mM}$ $\mathrm{KCl}, 5 \mathrm{mM} \mathrm{Na} \mathrm{HPO}_{4}-7 \mathrm{H}_{2} \mathrm{O}, 1.4 \mathrm{mM} \mathrm{KH_{2 }} \mathrm{PO}_{4}$, and $0.1 \%$ Tween; $\mathrm{pH}$ 7.4) (PBS-T), and incubated overnight at $4{ }^{\circ} \mathrm{C}$ with anti-c-myc antibody (Invitrogen) at 1:2500 or anti- $\alpha$-tubulin antibody (Sigma-Aldrich) at 1:10,000. Blots were then 
washed in PBS-T, and incubated for $1 \mathrm{~h}$ at RT with goat antimouse IgGs coupled to horseradish peroxidase (Dako), at 1:5000 dilution. Protein bands were detected by ECL chemiluminescence (Amersham GE Healthcare). The expression of VNUT was standardized by the expression of $\alpha$-tubulin of the same experiment. Images were captured with ImageQuant LAS 500 (GE Healthcare Life Sciences) and analyzed using ImageQuant TL.

\section{ATP release measurement}

ATP release was measured using ENLITEN ${ }^{\circledR}$ rLuciferase/Luciferin reagent (Promega). Culture medium $(100 \mu \mathrm{l})$ was collected under various experimental conditions and centrifuged at $600 \times \mathrm{g}$ for $5 \mathrm{~min}$ at $4{ }^{\circ} \mathrm{C}$, and $10 \mu \mathrm{l}$ aliquots of supernatant were transferred to wells of a 96-well plate placed on ice. Before the start of the experiments, $\mathrm{N} 2 \mathrm{a}$ cells were bathed in $\mathrm{Mg}^{2+}$-free Locke's buffer supplemented with $100 \mu \mathrm{M}$ ARL 67156, a

a

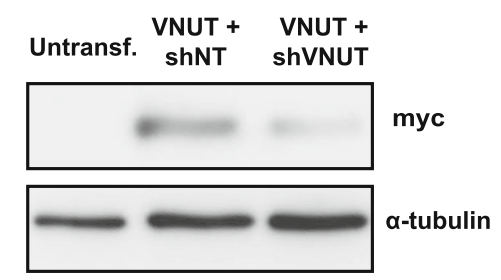

C

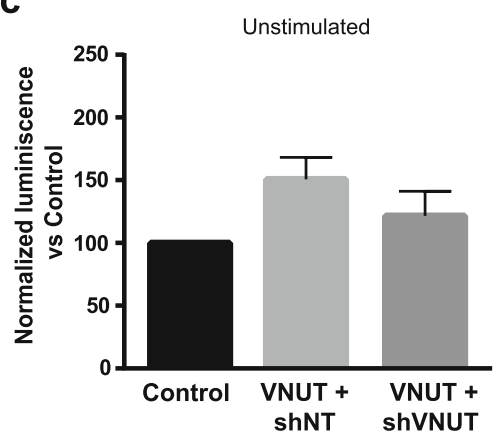

Fig. 2 Expression of a functional VNUT in N2a cells after differentiation with retinoic acid. a Western blotting of untransfected or transfected differentiated N2a cells with VNUT-myc after a 5-day differentiation process with retinoic acid $(10 \mu \mathrm{M})$. b qPCR of VNUT mRNA levels of differentiated N2a cells transfected with different plasmids. N2a cells were transfected with control plasmid, VNUT-myc + shNT or VNUTmyc $+\operatorname{shVNUT}$ as indicated. The values represent the mean \pm SEM $(n=$ $3, * * P<0.01, * * * P<0.001$, ANOVA with the Tukey's multiple comparisons test) normalized by the content of GAPDH transcript. c
Fig. 3 Expression of VNUT decreases neuritogenesis in differentiated N2a cells. a Representative confocal images of transfected N2a cells with control (RFP) (upper panels) or VNUT-myc (lower panels). Transfected control cells were identified by RFP fluorescence and cells expressing VNUT by anti-c-myc antibody ( $(r e d)$. N2a cells were immunostained with anti- $\beta$-III tubulin antibody (green) for the morphological analysis. Nuclei were counterstained with DAPI. Scale bar: $25 \mu \mathrm{m}$. b Percentage of transfected N2a cells without neurites or with neurite length lower than soma diameter $(<\mathrm{SD}, 10 \mu \mathrm{m})$, or higher than once $\mathrm{SD}(>1 \mathrm{SD}, 10 \mu \mathrm{m})$ or twice SD $(>2 \mathrm{SD}, 20 \mu \mathrm{m})$. N2a transfected with control RFP are represented by black bars and N2a transfected with VNUT by gray bars $(n=6, * P<0.05, * * P<0.01$, ANOVA with Sidak's multiple comparisons test). c Boxplot representation of the main neurite length in micrometer. Wiskers contains $10-90 \%$ of total measures $(* * * P>0.001$ with Kolmogorov-Smirnov analysis on the cumulative frequency). d Percentage of transfected N2a cells with different number of neurites. N2a transfected with RFP are represented by black bars and N2a transfected with VNUT by gray bars. Data show the mean \pm SEM of 70 cells $(n=6, * P<0.05, * * P<0.01$, ANOVA with Sidak's multiple comparisons test)

competitive inhibitor of ecto-ATPases [20] for $30 \mathrm{~min}$ at $37^{\circ} \mathrm{C}$ and the medium was collected to measure basal

b

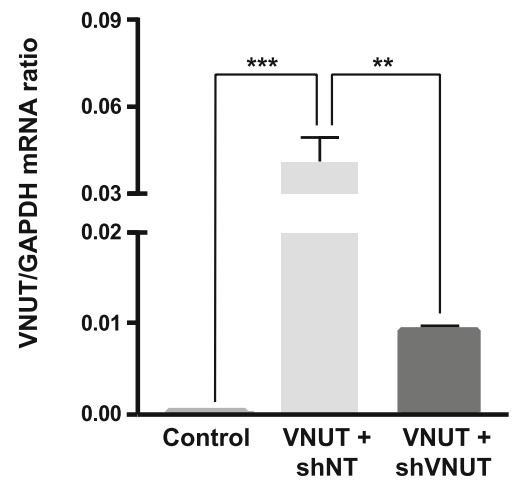

d

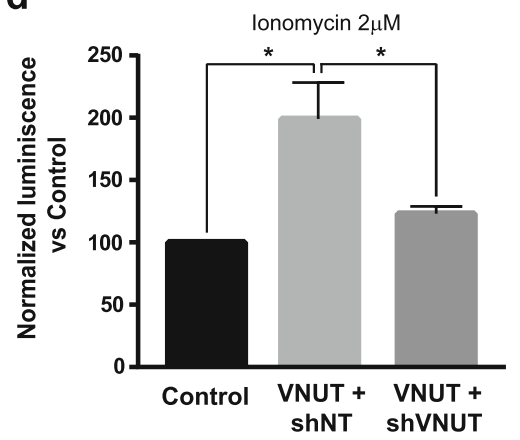

Luminescence activity of ATP release to the extracellular medium by transfected N2a cells without any stimulation. Luminescence was normalized against control cells. The values represent the mean \pm SEM ( $n=3$, non-significant, ANOVA with the Tukey's multiple comparisons test). $\mathbf{d}$ Luminescence activity of ATP release to the extracellular medium by transfected N2a cells after stimulation with $\mathrm{Ca}^{2+}$ ionophore ionomycin $(2 \mu \mathrm{M})$. Luminescence was normalized against control cells. The values represent the mean \pm SEM $(n=3, * * P<0.01$, ANOVA with the Tukey's multiple comparisons test) 


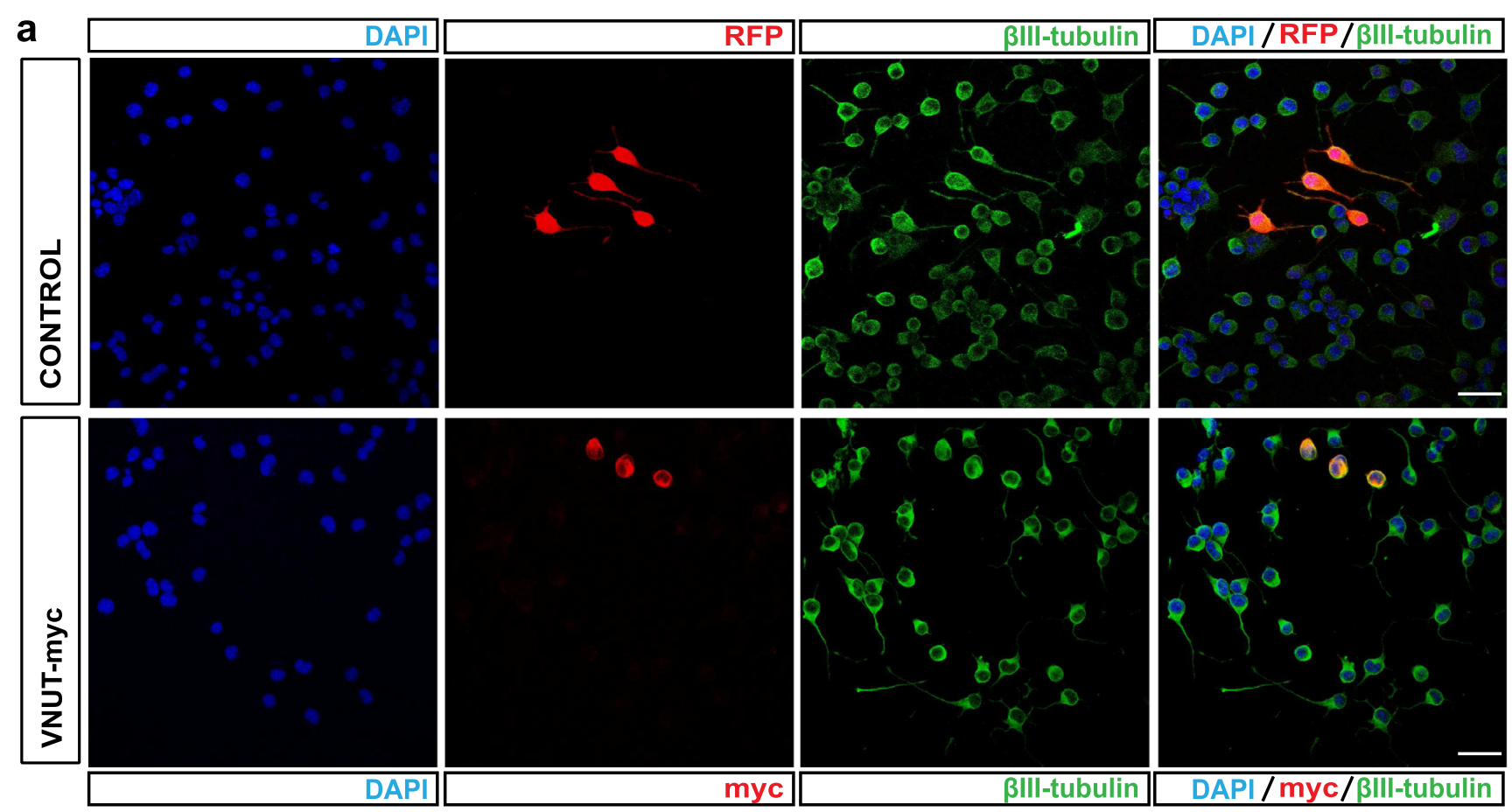

b

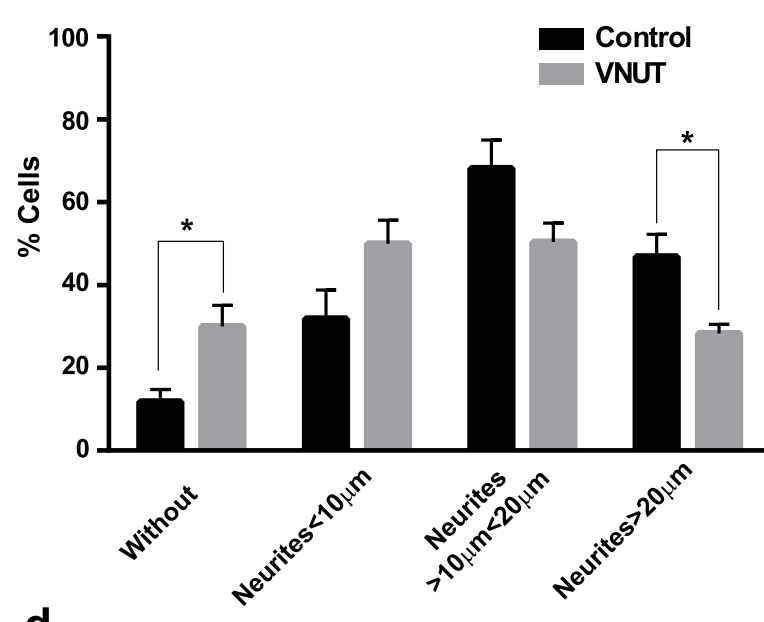

d

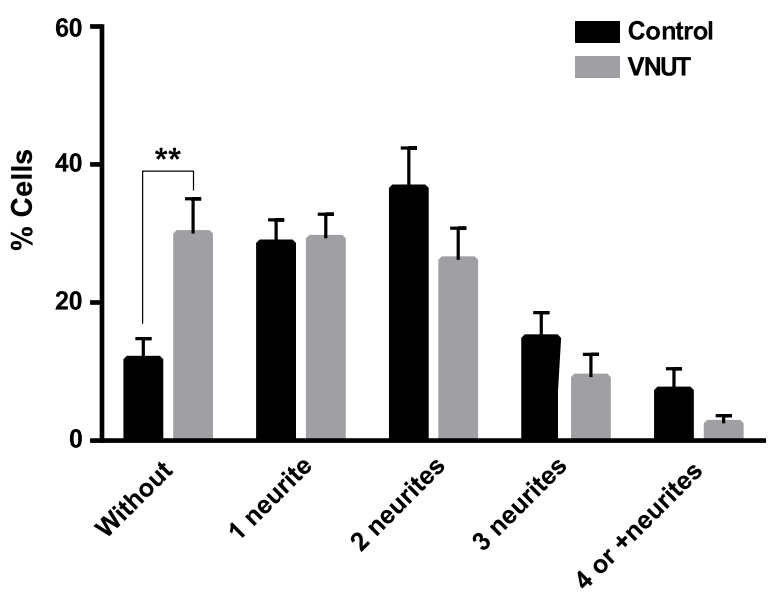

c

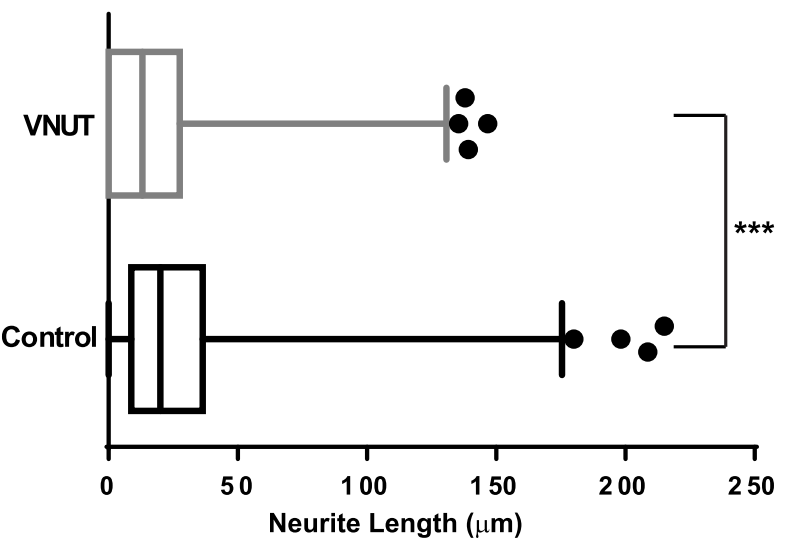


ATP. Then, cells were stimulated by adding ionomycin ( $2 \mu \mathrm{M}$ final concentration). Five minutes later, extracellular medium was again collected to measure evoked ATP concentration. The 96-well plate was set in a FLUOstar OPTIMA Microplate Luminometer (BMG LABTECH GmbH), and $100 \mu \mathrm{l}$ of rLuciferase/Luciferin reagent was automatically injected into each well at RT $\left(25{ }^{\circ} \mathrm{C}\right)$.

\section{Immunocytochemistry}

N2a-transfected cells cultured on coverslips placed in 35$\mathrm{mm}$ dishes were fixed with $4 \%$ PFA for $15 \mathrm{~min}$ and rinsed with PBS twice for $10 \mathrm{~min}$. Afterwards, cells on coverslips were permeabilized in blocking solution (Triton X-100 $0.1 \%$, FBS $5 \%$, and BSA $10 \%$ in PBS) for $1 \mathrm{~h}$ at RT. This was followed by incubation with primary antibodies: anti-c-myc (1:200), anti- $\beta$-III tubulin (1:1000) (Promega) and anti-synaptophysin (1:200) (Synaptic Systems). Subsequently, cells were washed with PBS three times and incubated for $1 \mathrm{~h}$ at RT with Alexa Fluor $594{ }^{\circledR}$ donkey anti-rabbit, Alexa Fluor $488^{\circledR}$ goat anti-mouse, and Alexa Fluor $647^{\circledR}$ goat anti-mouse (all from Invitrogen). Nuclei were counterstained with 4',6diamidino-2-phenylindole (DAPI, Invitrogen), a fluorescent stain that binds strongly to DNA. Coverslips were mounted on glass slides using FluoroSave ${ }^{\mathrm{TM}}$ Reagent (Calbiochem). Images were acquired using a confocal laser microscope (Leica TCS SPE) using $20 \times$ W/IR objective lenses. The analysis of neurite length and ramifications was carried out using Image J v1.49j (NIH) and Neuron J plugin v1.4.2 [21]. Neurite outgrowth was quantified by determining the percentage of cells that present neurites once or twice longer than the soma diameter (SD) as described by Ravichandra and Joshi (1999) [22]. Ten different fields in the culture dishes containing 70 cells in each field were randomly selected and counted. The mean percentage of neurite-bearing cells was obtained for each culture. Each assay was repeated six times in three independent cultures.

\section{Statistical analysis}

Data are shown as mean values \pm standard error of the mean (SEM). All experiments shown were reproduced 3-6 independent times. Figures and statistical analyses were generated using GraphPad Prism 6 (GraphPad Software). Results were analyzed by unpaired Student's $t$ test or ANOVA with Tukey or Sidak's multiple comparisons test as indicated. Neurite length was compared using Kolmogorov-Smirnov analysis on the cumulative frequency distribution. The statistical test used and $P$ values
Fig. 4 Knockdown of VNUT expression increases neuritogenesis in differentiated N2a cells. a Representative confocal images of N2a cells co-transfected with VNUT-myc and either shNT (upper panels) or shVNUT (lower panels). Transfected cells were identified by GFP fluorescence (blue). N2a cells were immunostained with anti-c-myc (red) and anti- $\beta$-III tubulin (green) antibodies. Scale bar: $25 \mu \mathrm{m}$. b Percentage of transfected N2a cells without or with neurite length lower than soma diameter $(<\mathrm{SD}, 10 \mu \mathrm{m})$ or higher than once SD $(>1 \mathrm{SD}, 10 \mu \mathrm{m})$ or twice SD (>2SD. $20 \mu \mathrm{m})$. N2a transfected with $\mathrm{VNUT}^{+}$shNT are represented by black bars and N2a transfected with $\mathrm{VNUT}^{+}$shVNUT by gray bars $\left(n=6,{ }^{*} P<0.05,{ }^{*} P<0.01\right.$, ANOVA with Sidak's multiple comparisons test). $\mathbf{c}$ Boxplot representation of the main neurite length in micrometer. Wiskers contains $10-90 \%$ of total measures $(* * P>0.01$ with Kolmogorov-Smirnov analysis on the cumulative frequency). d Percentage of transfected N2a cells with different number of neurites. $\mathrm{N} 2 \mathrm{a}$ transfected with $\mathrm{VNUT}^{+}$shNT are represented by black bars and N2a transfected with $\mathrm{VNUT}^{+}$shVNUT by gray bars. Data show the mean \pm SEM of 70 cells $(n=6, * P<0.05, * * P<0.01$, ANOVA with Sidak's multiple comparisons test)

are both indicated in each figure legend. $P \leq 0.05$ was considered statistically significant.

\section{Results}

\section{Design of molecular tools for VNUT expression}

In order to validate the molecular tools used in this study, N2a cells were transfected with the designed constructs VNUTmyc (Fig. 1a) and shRNAs either control (shNT) or specifically designed against VNUT (shVNUT). After $24 \mathrm{~h}$, the transfection efficiency was about $85 \%$ and mRNA and protein levels of VNUT were analyzed by qPCR (Fig. 1b) and Western blotting (Fig. 1c), respectively. These experiments demonstrated that VNUT is successfully expressed in transfected N2a cells. Moreover, the expression of this transporter was reduced when N2a cells were co-transfected with VNUT-myc and shVNUT (Fig. 1b, c). Likewise, immunocytochemistry assays were performed incubating transfected N2a cells with anti-c-myc antibody, and a vesicular colocalization with vesicular marker synaptophysin could be observed in confocal fluorescence images (Fig. 1d).

\section{Retinoic acid-induced N2a differentiation keeps VNUT overexpression in transfected cells}

Transfected N2a cells were submitted to retinoic acid differentiation according to Tremblay et al. (2010) [19]. Western blotting and qPCR assays were performed to confirm that expression of VNUT is maintained in differentiated N2a cells. After 5 days with retinoic acid, VNUT mRNA and protein levels were analyzed. The expression of the transporter was detected in differentiated N2a cells co-transfected with VNUT and shNT by Western blotting (Fig. 2a) and qPCR (Fig. 2b) assays. 


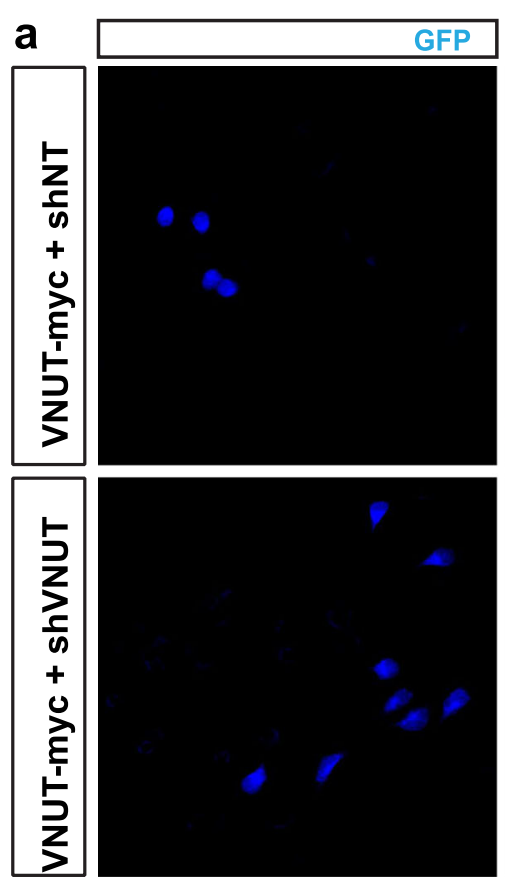

b

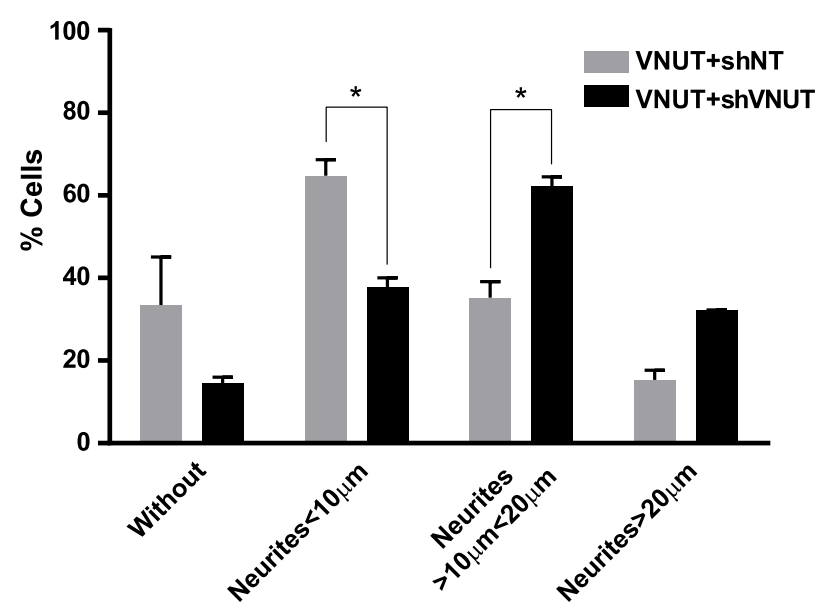

d

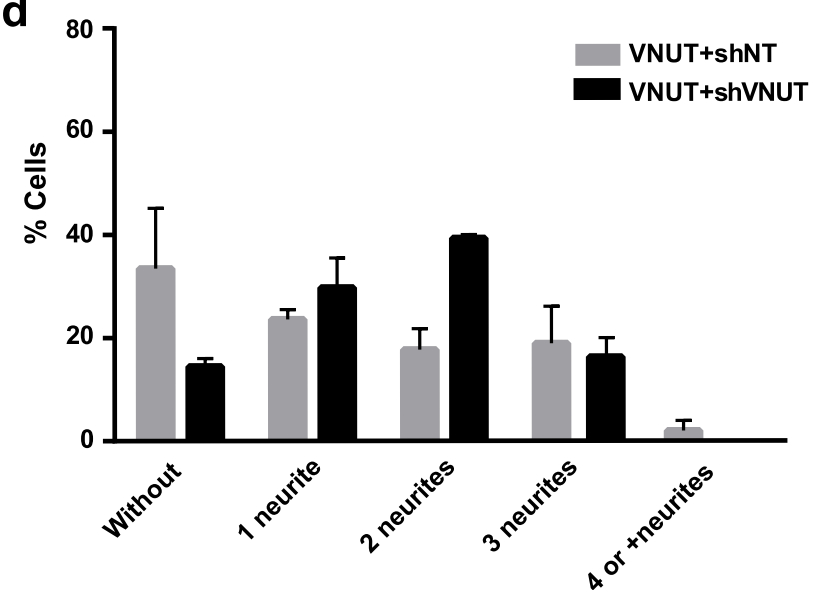

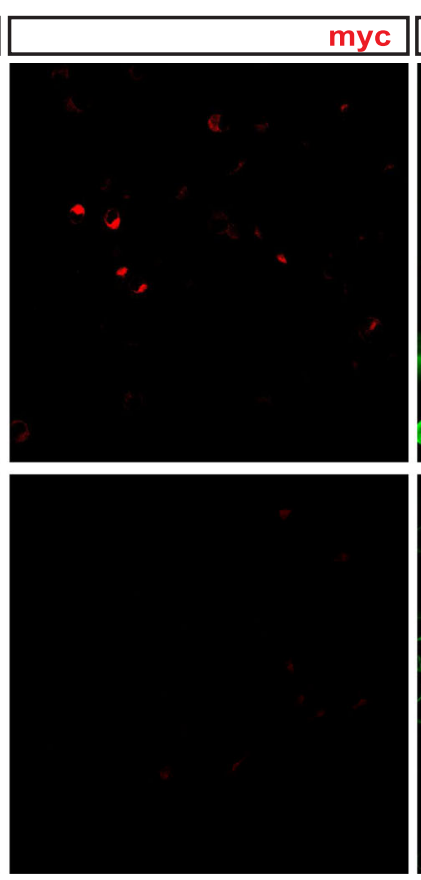
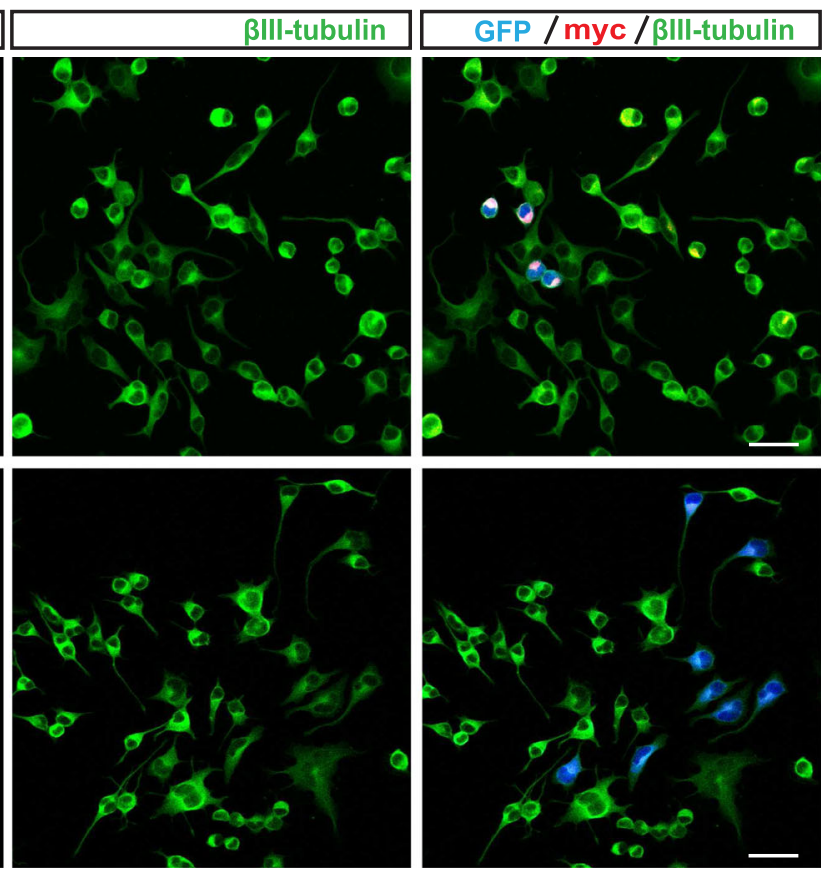

C

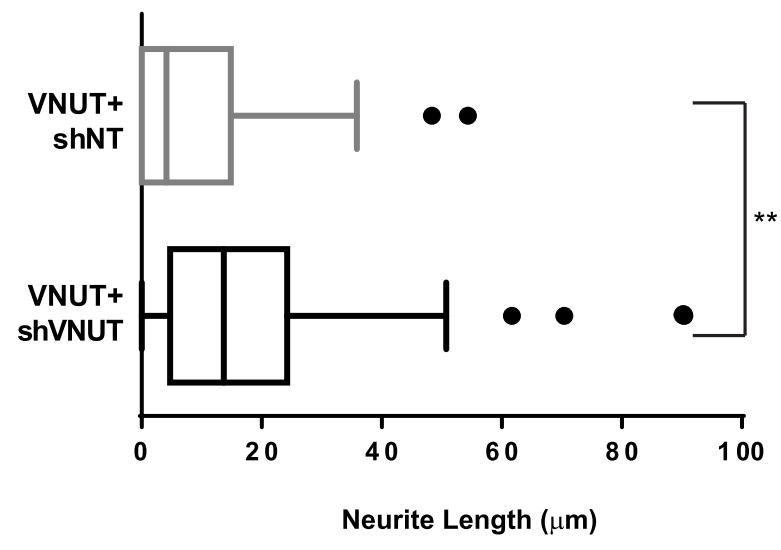


The reduction on the expression of VNUT after 5 days of differentiation, compared with cells freshly transfected, was caused by the loss of transfected cells associated to transient transfections, where only about $15 \%$ of cells remain transfected. On the other hand, VNUT mRNA levels were reduced in differentiated N2a cells cotransfected with VNUT and shVNUT (Fig. 2a, b). Furthermore, the functionality of the vesicular transporter was confirmed by using the luciferin-luciferase assay to quantify the luminescence signal produced by the released ATP (Fig. 2c). Previous studies demonstrated that $\mathrm{N} 2 \mathrm{a}$ cells have a mechanism of ATP release depending on the increase in intracellular $\left[\mathrm{Ca}^{2+}\right]$, involving the exocytotic machinery [16]. Differentiated N2a cells were stimulated with $\mathrm{Ca}^{2+}$-selective ionophore, ionomycin $2 \mu \mathrm{M}$, for $5 \mathrm{~min}$ and extracellular medium was collected with or without stimulation. Cells co-transfected with VNUT and shNT showed the highest values of luminescence, which decreased when co-transfected with VNUT and shVNUT (Fig. 2c, d). These results indicate that the vesicular nucleotide transporter is functional when it was expressed in differentiated N2a cells and kept functional during retinoic acid differentiation process.

\section{Neuritogenesis is reduced in N2a overexpressing VNUT}

After verification that VNUT is functional and its expression remains in differentiated $\mathrm{N} 2 \mathrm{a}$ cells, we wanted to analyze the possible effects that expression of VNUT could produce in the neuritogenesis of these cells. To study the probable morphological changes, N2a cells were transfected either with control or VNUT-myc construct, and identified by immunostaining with anti-c-myc antibody (for VNUT-myc-transfected cells) or RFP fluorescence from pRFP-C-RS vector as transfection control (Fig. 3a). This vector has a red fluorescent protein (RFP)-coding sequence which allows to identify transfected cells (Fig. 3a). A specific anti- $\beta$-III tubulin antibody was used to observe the neuronal morphology (Fig. 3a) and go ahead with the quantification of neurites.

In N2a cells that expressed VNUT the percentage of cells without neurites was significantly increased compared to control $(30.0 \% \pm 5.0$ versus $11.8 \% \pm 2.9)$ (Fig. 3b and d). Similarly, the expression of VNUT decreased the median of length of the principal neurite $13.07 \mu \mathrm{m}$ for VNUT transfected cells compared with $20.00 \mu \mathrm{m}$ for control cells (Fig. 3c) $(P<0.0001$ with Kolmogorov-Smirnov analysis on the cumulative frequency distribution). In addition, if a distribution of cells is made accordingly to neurite length, the expression of VNUT increases the percentage of cells with neurites shorter than the soma diameter $(\mathrm{SD} 10 \mu \mathrm{m})(49.9 \pm$
Fig. 5 VNUT is a component of the purinergic machinery required for control of axonal growth and neuritogenesis. ATP is stored in secretory vesicles through VNUT; this transporter uses the generated gradient $(\Delta$ $\left.\mu_{\mathrm{H}+}\right)$ by V-ATPase to translocate ATP into vesicles. ATP is released to extracellular medium through calcium-dependent mechanism. Once released, ATP can activate purinergic receptors, such as $\mathrm{P} 2 \mathrm{X} 7$, in an autocrine manner that results in various physiological cellular events. Simultaneously, ATP can be degraded to its metabolites by ectonucleotidases activity. a The abundant presence of extracellular ATP induces P2X7 activation, and the intracellular $\mathrm{Ca}^{2+}$ increases results on axonal length and neuritogenesis reduction in this cellular model [16]. b On the contrary, a decrease or destruction of extracellular ATP results in an increase of axonal length and branching

$5.7 \%$ versus $31.9 \pm 6.9 \%$ ) (Fig. 3b). Otherwise, the percentage of cells with neurites longer than SD $(>10 \mu \mathrm{m})$ $(50.4 \pm 4.5 \%$ versus $68.1 \pm 6.9 \%)$ and neurites twice longer than SD $(>20 \mu \mathrm{m})(28.3 \pm 2.2 \%$ versus $46.8 \pm 5.5 \%)$ decreased in those cells that expressed VNUT (Fig. 3b). In the same way, expression of VNUT decreased the percentage of cells with two $(26.2 \pm 4.5 \%$ versus $36.6 \pm$ $5.8 \%)$, three $(9.2 \pm 3.3 \%$ versus $14.9 \pm 3.9 \%)$, and four or more $(2.5 \pm 1.1 \%$ versus $7.3 \pm 3.1 \%)$ neurites compared to control cells (Fig. 3c).

\section{VNUT knockdown restores differentiation process in $\mathrm{N} 2 \mathrm{a}$ cell line}

In order to corroborate that the observed effect in the differentiation of $\mathrm{N} 2 \mathrm{a}$ cells is due to expression of the vesicular transporter, shVNUT was used to knockdown its expression. N2a cells were co-transfected with VNUT-myc and either shNT (control) or shVNUT. Cells co-transfected with both plasmids were identified by shRNA-GFP fluorescence emission and anti-myc immunolabeling addressed to detect VNUT-myc (Fig. 4a). The knockdown of VNUT increased the median of length of the principal neurite, from $9.1 \mu \mathrm{m}$ for VNUTand shNT cells to $18.3 \mu \mathrm{m}$ for VNUT and shVNUT transfected cells (Fig. 4c) $(P<0.001$ with KolmogorovSmirnov analysis on the cumulative frequency distribution). The knockdown of VNUT expression decreased the percentage of cells without neurites ( $33.4 \pm 11.7 \%$ to $14.5 \pm 1.5 \%$ ) (Fig. 4b). Likewise, the same effect was observed in cells with neurites shorter than SD $(<10 \mu \mathrm{m})$ whose percentage significantly decreased (64.7 $\pm 3.9 \%$ to $37.7 \pm 2.3 \%$ ) (Fig. 4 c). However, the percentages of cells with both neurites longer than SD $(>10 \mu \mathrm{m})(35.2 \pm 3.8 \%$ to $62.3 \pm 2.3 \%)$ (Fig. $4 \mathrm{c})$ and neurites twice as long as SD $(>20 \mu \mathrm{m})(15.3 \pm 2.3 \%$ to 32.1 $\pm 0.1 \%$ ) were increased compared to N2a cells co-transfected with VNUT and shNT. Regarding the number of neurites, the effect of VNUT knockdown was not as evident as in the observed neurite outgrowth (Fig. 4d). These results indicate that the reduction of VNUT expression increases neuritogenesis in differentiated N2a cells. 

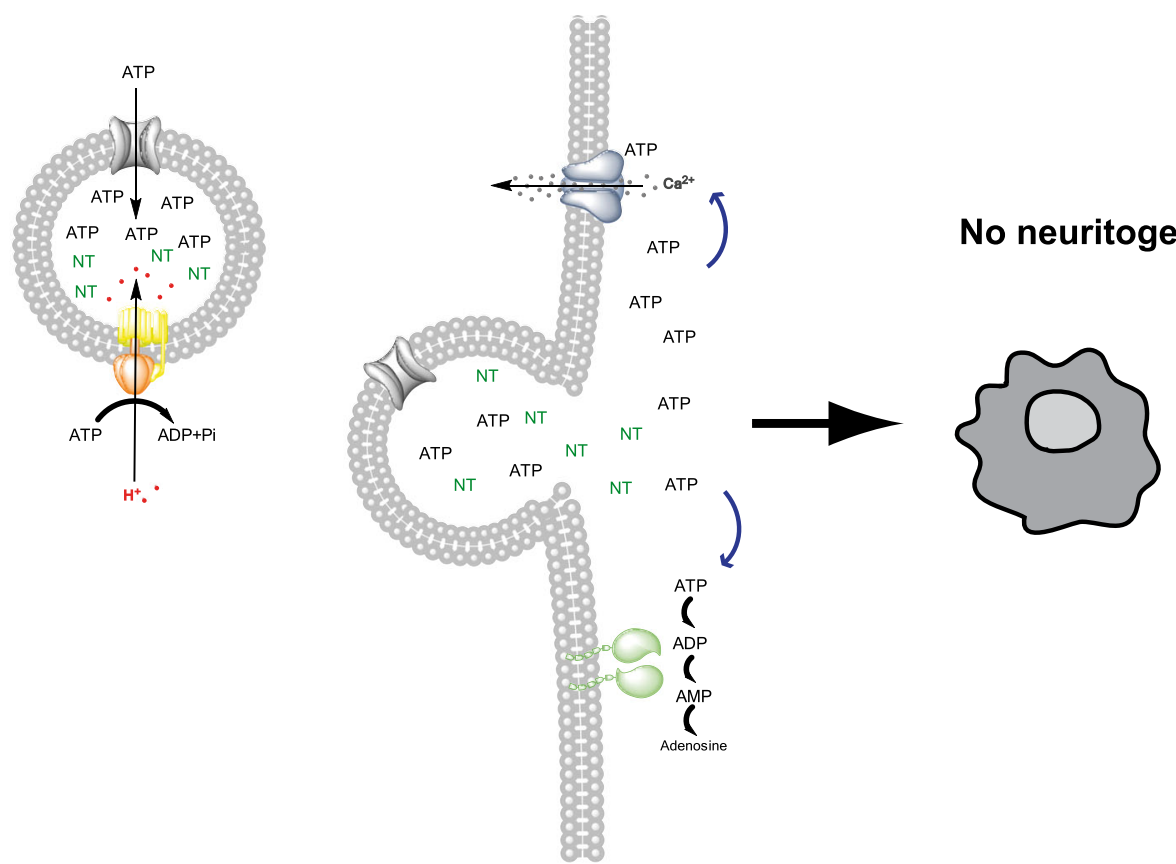

b
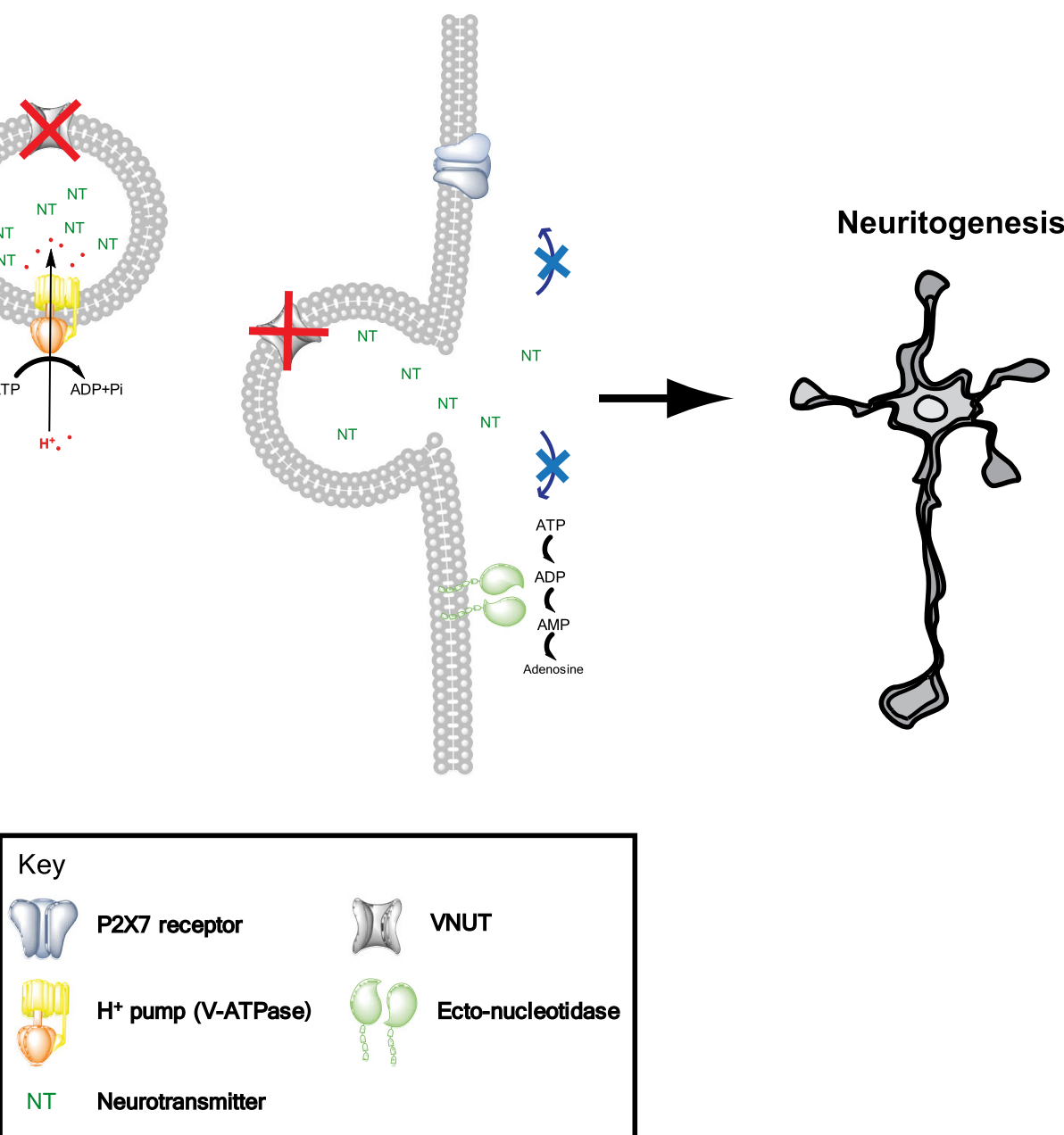


\section{Discussion}

The aim of this experimental work was to demonstrate that the vesicular nucleotide transporter (VNUT) is a key component required to accomplish the cellular biological events involving extracellular ATP in neurosecretory cells. Nucleotide P2X and P2Y receptors are present in most mammalian cells, and their physiological or pathological signaling are unveiled step by step $[9,23,24]$. For secretory cells, the cloning and characterization of the elusive VNUT opened new possibilities of understanding the ATP extracellular cycle and its relation with secretory tissues-related diseases $[8$, 25-27]. The first tissues studied were from neural or neuroendocrine origin, and the presence of VNUT was demonstrated by immunohistochemistry and RNA in situ hybridization [28-30]. It is worth emphasizing that synaptic vesicles and chromaffin cell granules are able to store ATP and other nucleotides up to $0.1 \mathrm{M}$, among them are the diadenosine polyphosphates which are agonists on several P2X and P2Y receptors [31].

Neuroblastoma N2a cell line has proved to be a valuable model to analyze cell biology events and consequently selected to understand the role of VNUT in neural differentiation events $[14,15]$. A simplified scheme of this process is shown in Fig. 5. On the other hand, these cells express very small amounts of endogenous VNUT, which was a requisite for better understanding of the expression effects [16]. Expression of the VNUT in secretory cells has already been reported in PC12 cells, a chromaffin cell model, being able to increase ATP secretion in depolarizing conditions [8]. In our model, expression of VNUT co-localizes with the vesicular marker synaptophysin, and as reported for PC12 cell line, the membrane depolarization increases the ATP release. These results are important experimental facts to consider VNUT as a functional transporter. Furthermore, sustained expression of VNUT is observed when N2a cells are subjected to retinoic acid differentiation, this being a necessary condition to evaluate the ATP effects on neuritogenesis and differentiation.

In this regard, expression of VNUT clearly reduces the neuritogenic processes in N2a cells compared to control cells. Changes induced in the cellular morphology allow a precise quantification and comparison of length of neurite processes in VNUT expressing and non-expressing cells. Additional experiments to corroborate the VNUT negative effect on neuritogenic processes required N2a cells co-transfected with VNUT and a knockdown, shVNUT. These cells, where VNUT expression was reduced, recovered their morphology with more prominent neuritogenic processes. This confirms the hypothesis of VNUT is involved in neuronal differentiation as part of a mechanism requiring extracellular ATP acting through P2X7 receptor at the axonal growth cone [32, 14, 17]. The physiological relevance and future perspectives of VNUT as a pharmacological target derive from its capacity to increase extracellular ATP levels. ATP vesicular release has also been reported from the mucin granules of airway epithelial cells, microglia, and a large variety of neuroendocrine secretory tissues $[33,25,30]$. The physiopathological role of extracellular ATP and its exocytotic release in many tissues supports VNUT as a new and relevant pharmacological target.

Acknowledgments This work has been supported by research grants from Ministerio de Ciencia e Innovación (BFU2011-24743), the Spanish Ion Channel Initiative (CSD2008-00005), Fundación Marcelino Botín and Comunidad de Madrid S2013/ICE-2958 BRADE-CM.

Conflict of interest The authors declare that there are no conflicts of interest, financial or otherwise.

Open Access This article is distributed under the terms of the Creative Commons Attribution 4.0 International License (http://creativecommons. org/licenses/by/4.0/), which permits unrestricted use, distribution, and reproduction in any medium, provided you give appropriate credit to the original author(s) and the source, provide a link to the Creative Commons license, and indicate if changes were made.

\section{References}

1. Schuldiner S, Shirvan A, Linial M (1995) Vesicular neurotransmitter transporters: from bacteria to humans. Physiol Rev 75(2):369392

2. Van Liefferinge J, Massie A, Portelli J, Di Giovanni G, Smolders I (2013) Are vesicular neurotransmitter transporters potential treatment targets for temporal lobe epilepsy? Front Cell Neurosci 7:139. doi:10.3389/fncel.2013.00139

3. Li H, Harlow ML (2014) Individual synaptic vesicles from the electroplaque of Torpedo californica, a classic cholinergic synapse, also contain transporters for glutamate and ATP. Physiol Rep 2(1): e00206. doi:10.1002/phy2.206

4. Gualix J, Alvarez AM, Pintor J, Miras-Portugal MT (1999) Studies of chromaffin granule functioning by flow cytometry: transport of fluorescent epsilon-ATP and granular size increase induced by ATP. Recept Channels 6(6):449-461

5. Burnstock G (2004) Cotransmission. Curr Opin Pharmacol 4(1): 47-52. doi:10.1016/j.coph.2003.08.001

6. Weber A, Westhead EW, Winkler H (1983) Specificity and properties of the nucleotide carrier in chromaffin granules from bovine adrenal medulla. Biochem J 210(3):789-794

7. Gualix J, Abal M, Pintor J, Garcia-Carmona F, Miras-Portugal MT (1996) Nucleotide vesicular transporter of bovine chromaffin granules. Evidence for a mnemonic regulation. J Biol Chem 271(4): 1957-1965

8. Sawada K, Echigo N, Juge N, Miyaji T, Otsuka M, Omote H, Yamamoto A, Moriyama Y (2008) Identification of a vesicular nucleotide transporter. Proc Natl Acad Sci U S A 105(15):56835686. doi:10.1073/pnas.0800141105

9. Burnstock G (2008) Purinergic signalling and disorders of the central nervous system. Nat Rev Drug Discov 7(7):575-590. doi:10. $1038 /$ nrd2605

10. Abbracchio MP, Burnstock G, Verkhratsky A, Zimmermann H (2009) Purinergic signalling in the nervous system: an overview. Trends Neurosci 32(1):19-29. doi:10.1016/j.tins.2008.10.001 
11. Zimmermann H (2006) Ectonucleotidases in the nervous system. Novartis Found Symp 276:113-128, discussion 128-130, 233-117, 275-181

12. Robson SC, Sevigny J, Zimmermann H (2006) The E-NTPDase family of ectonucleotidases: structure function relationships and pathophysiological significance. Purinergic Signal 2(2):409-430. doi:10.1007/s11302-006-9003-5

13. Gomez-Villafuertes R, Pintor J, Miras-Portugal MT, Gualix J (2014) Ectonucleotide pyrophosphatase/phosphodiesterase activity in Neuro-2a neuroblastoma cells: changes in expression associated with neuronal differentiation. J Neurochem. doi:10.1111/jnc.12794

14. Gomez-Villafuertes R, del Puerto A, Diaz-Hernandez M, Bustillo D, Diaz-Hernandez JI, Huerta PG, Artalejo AR, Garrido JJ, MirasPortugal MT (2009) Ca2+/calmodulin-dependent kinase II signalling cascade mediates $\mathrm{P} 2 \mathrm{X} 7$ receptor-dependent inhibition of neuritogenesis in neuroblastoma cells. FEBS J 276(18):53075325. doi:10.1111/j.1742-4658.2009.07228.x

15. Wu PY, Lin YC, Chang CL, Lu HT, Chin CH, Hsu TT, Chu D, Sun SH (2009) Functional decreases in P2X7 receptors are associated with retinoic acid-induced neuronal differentiation of Neuro-2a neuroblastoma cells. Cell Signal 21(6):881-891

16. Gutierrez-Martin Y, Bustillo D, Gomez-Villafuertes R, SanchezNogueiro J, Torregrosa-Hetland C, Binz T, Gutierrez LM, MirasPortugal MT, Artalejo AR (2011) P2X7 receptors trigger ATP exocytosis and modify secretory vesicle dynamics in neuroblastoma cells. J Biol Chem 286(13):11370-11381. doi:10.1074/jbc.M110. 139410

17. Diez-Zaera M, Diaz-Hernandez JI, Hernandez-Alvarez E, Zimmermann H, Diaz-Hernandez M, Miras-Portugal MT (2011) Tissue-nonspecific alkaline phosphatase promotes axonal growth of hippocampal neurons. Mol Biol Cell 22(7):1014-1024. doi:10. 1091/mbc.E10-09-0740

18. Reynolds A, Leake D, Boese Q, Scaringe S, Marshall WS, Khvorova A (2004) Rational siRNA design for RNA interference. Nat Biotechnol 22(3):326-330. doi:10.1038/nbt936

19. Tremblay RG, Sikorska M, Sandhu JK, Lanthier P, RibeccoLutkiewicz M, Bani-Yaghoub M (2010) Differentiation of mouse Neuro 2A cells into dopamine neurons. J Neurosci Methods 186(1): 60-67. doi:10.1016/j.jneumeth.2009.11.004

20. Levesque SA, Lavoie EG, Lecka J, Bigonnesse F, Sevigny J (2007) Specificity of the ecto-ATPase inhibitor ARL 67156 on human and mouse ectonucleotidases. Br J Pharmacol 152(1):141-150. doi:10. 1038/sj.bjp.0707361

21. Meijering E, Jacob M, Sarria JC, Steiner P, Hirling H, Unser M (2004) Design and validation of a tool for neurite tracing and analysis in fluorescence microscopy images. Cytometry Part A: J Int Soc Anal Cytol 58(2):167-176. doi:10.1002/cyto.a.20022
22. Ravichandra B, Joshi PG (1999) Regulation of transmembrane signaling by ganglioside GM1: interaction of anti-GM1 with Neuro2a cells. J Neurochem 73(2):557-567

23. Tsuda M, Masuda T, Tozaki-Saitoh H, Inoue K (2013) P2X4 receptors and neuropathic pain. Front Cell Neurosci 7:191. doi:10.3389/ fncel.2013.00191

24. Salter MW, Beggs S (2014) Sublime microglia: expanding roles for the guardians of the CNS. Cell 158(1):15-24. doi:10.1016/j.cell. 2014.06.008

25. Sesma JI, Kreda SM, Okada SF, van Heusden C, Moussa L, Jones LC, O'Neal WK, Togawa N, Hiasa M, Moriyama Y, Lazarowski ER (2013) Vesicular nucleotide transporter regulates the nucleotide content in airway epithelial mucin granules. Am J Physiol Cell Physiol 304(10):C976-C984. doi:10.1152/ajpcell.00371.2012

26. Geisler JC, Corbin KL, Li Q, Feranchak AP, Nunemaker CS, Li C (2013) Vesicular nucleotide transporter-mediated ATP release regulates insulin secretion. Endocrinology 154(2):675-684. doi:10. 1210/en.2012-1818

27. Perez de Lara MJ, Pintor J (2014) Presence and release of ATP from the retina in an Alzheimer's disease model. J Alzheimer's Dis JAD. doi:10.3233/JAD-141005

28. Larsson M, Sawada K, Morland C, Hiasa M, Ormel L, Moriyama Y, Gundersen V (2012) Functional and anatomical identification of a vesicular transporter mediating neuronal ATP release. Cereb Cortex 22(5):1203-1214. doi:10.1093/cercor/bhr203

29. Nishida K, Nomura Y, Kawamori K, Moriyama Y, Nagasawa K (2014) Expression profile of vesicular nucleotide transporter (VNUT, SLC17A9) in subpopulations of rat dorsal root ganglion neurons. Neurosci Lett 579C:75-79. doi:10.1016/j.neulet.2014.07. 017

30. Haanes KA, Kowal JM, Arpino G, Lange SC, Moriyama Y, Pedersen PA, Novak I (2014) Role of vesicular nucleotide transporter VNUT (SLC17A9) in release of ATP from AR42J cells and mouse pancreatic acinar cells. Purinergic Signal. doi:10.1007/ s11302-014-9406-7

31. Gualix J, Fideu MD, Pintor J, Rotllan P, Garcia-Carmona F, MirasPortugal MT (1997) Characterization of diadenosine polyphosphate transport into chromaffin granules from adrenal medulla. FASEB J Off Publ Fed Am Soc Exp Biol 11(12):981-990

32. Diaz-Hernandez M, del Puerto A, Diaz-Hernandez JI, Diez-Zaera M, Lucas JJ, Garrido JJ, Miras-Portugal MT (2008) Inhibition of the ATP-gated $\mathrm{P} 2 \mathrm{X} 7$ receptor promotes axonal growth and branching in cultured hippocampal neurons. J Cell Sci 121(Pt 22): 3717-3728. doi: $10.1242 /$ jcs. 034082

33. Imura Y, Morizawa $Y$, Komatsu R, Shibata K, Shinozaki Y, Kasai H, Moriishi K, Moriyama Y, Koizumi S (2013) Microglia release ATP by exocytosis. Glia 61(8):1320-1330. doi:10.1002/glia.22517 